\title{
THE NOVEMBER MEETING IN IOWA CITY
}

The five hundred eighth meeting of the American Mathematical Society was held in conjunction with a meeting of the Central Section of the Institute of Mathematical Statistics at the State University of Iowa on Friday, November 26, 1954. There were about 90 registrations, including 60 members of the Society.

Professor R. P. Boas presided at an address delivered by Professor Vaclav Hlavaty of Indiana University. The address was entitled Unified field theory and was given by invitation of the Committee to Select Hour Speakers for Western Sectional Meetings. There was a session for contributed papers in the afternoon presided over by Professor H. T. Muhly.

Abstracts of the papers presented follow. Those having the letter " $t$ " after their numbers were read by title.

\section{Algebra and Theory of Numbers}

130. H. W. Becker: A case history of the impotence of abstract algebra in Diophantine analysis.

A Pythagorean tetrahedron has $t, u, v, y, x, z=\left(\alpha^{2}+\beta^{2}\right)\left(\gamma^{2}+\delta^{2}\right) \pm 4 \alpha \beta \gamma \delta, 2(\beta \gamma+\alpha \delta)$ $(\alpha \gamma \pm \beta \delta),\left(\alpha^{2}-\beta^{2}\right)\left(\gamma^{2}-\delta^{2}\right), 4\left[\alpha \beta \gamma \delta\left(\alpha^{2}+\beta^{2}\right)\left(\gamma^{2}+\delta^{2}\right)\right]^{1 / 2}$. In Dickson's History II, pp. $644-647, z$ is satisfied in large integers by $\alpha, \beta, \gamma, \delta=\Omega$, or else by (1) $\alpha \beta\left(\alpha^{2}+\beta^{2}\right)$ $=\gamma \delta\left(\gamma^{2} \div \delta^{2}\right)$. The 2 known solutions of (1), Euler ${ }^{188}(5)$ and Gerardin ${ }^{181 a}$, have duals on reversing alternate signs which satisfy (2) $a b c d\left(a^{2}-b^{2}\right)\left(c^{2}-d^{2}\right)=\square=y^{2} / 16$. In addition to these 2, "Je possede 3 formules de degres respectifs: 7, 13, 19."-M. Rignaux, L'Int. des Math. vol. 26 (1919) p. 5, which 3 do not seem to have come to light. In the Petrus transform of $a, b, c, d$ (see Euler ${ }^{253}$, p. 661 ibid.): $\alpha, \beta, \gamma, \delta=k, l, j, i$ $=a^{2}-b^{2}, 2 a b, c^{2}-d^{2}, 2 c d$. The only known simple parametric solution for $z$ is $\alpha, \beta, \gamma, \delta$ $=\left(r^{2}-s^{2}\right)^{2}, 4 r s\left(r^{2}+s^{2}\right), r, s$, Euler's ${ }^{304}$ (ii) PT (ibid., p. 667-668), dual of Rolle's PT (ibid. p. 172, Fermat ${ }^{62 \mathrm{a}}$; p. 504, O'Riordan ${ }^{33}$, last line). But the simplest solutions are mavericks (as yet unformulated) found by tabulating $\alpha \beta\left(\alpha^{2}+\beta^{2}\right) / \square$. Of the 10 known solutions in integers $\leqq 35: 8,1,5,1 ; 9,8,5,2 ; 13,9,5,1 ; 13,9,8,1 ; 15,8,3,1$; $19,17,19,9 ; 20,9,13,4 ; 29,2,5,2 ; 29,2,9,8 ; 35,12,21,20$, only the last is not a maverick, an algebraic coverage or potency of only $10 \%$. If $a b\left(a^{2} \mp b^{2}\right) / \square=\alpha \beta\left(\alpha^{2}\right.$ $\left.\pm \beta^{2}\right) / 2 \square$, then $\alpha, \beta, 2 a b, a^{2} \mp b^{2}$ is a solution of $z$ or $y$, by tabular algebra. Thus from 9,4 and 5, 1 resp. (upper signs) one gets $\alpha, \beta, \gamma, \delta=5,1,72,65$. (Received October 13, 1954.)

131t. Ellen Correl and Melvin Henriksen: $A$ note on rings of bounded continuous functions with values in a division ring.

Let $C^{*}(X, A)$ denote the ring of all bounded, continuous $A$-valued functions on a topological space $X$ that is completely regular with respect to a topological division ring $A$ (for definitions and background, see Goldhaber and Wolk, Duke Math. J. vol. 21 (1954) pp. 565-569). Stone's theorem is said to hold for $A$ if $C^{*}(X, A) / M=A$ for every maximal ideal $M$ of $C^{*}(X, A)$. Theorem: Stone's theorem holds for locally compact 
division rings. Because of known structure theorems for locally compact division rings (Otobe, Jap. J. Math. vol. 19 (1945) pp. 189-202), this theorem is new only when $A$ is totally disconnected (in which case $X$ is zero-dimensional). Goldhaber and Wolk have shown (loc. cit.) that if $A$ is of type $V$ (in the sense of Kaplansky), then Stone's theorem holds for $A$ only if $A$ is locally sequentially compact. They also ask if all the maximal ideals of $C^{*}(X, A)$ are of a certain specified form (too complicated to be stated here). The answer to this question is in the negative, even if both $X$ and $A$ are the real field. (The second author was supported in part by the National Science Foundation, contract no. NSF-G1129.) (Received October 13, 1954.)

\section{W. G. Leavitt: Modules over rings of words.}

In this paper are considered finitely based modules over certain "word" rings of the type introduced by Malcev. Over the word ring itself (all sums with integral coefficients of words in a finite set of symbols) any finitely based module, while containing infinite independent sets, nevertheless has invariant basis number. This result also holds for Malcev's ring. Thus imbeddability in a division ring, while sufficient for invariance of basis number, is not necessary. It is shown, finally, that for any integer $n>1$ an integral domain is constructable over which a finitely based module has invariant basis number if and only if it has a basis of length $<n$. The basic ring used is a word ring $R$ in the symbols of $n$ by $(n+1)$ and $(n+1)$ by $n$ matrices $A$ and $B$. The set $H$ of all members of $R$ having form $x y z$, with $x, z \in R$ and $y$ an element of either $A B-I_{n+1}$ or $B A-I_{n}$, is shown to be a two-sided ideal, and the factor ring $K=R / H$ is found to be an integral domain of the desired type. (Received September 24, 1954.)

\section{Bernard Vinograde: $A$ property of the product of division alge- bras.}

Let $D_{1}$ and $D_{2}$ be division algebras of finite dimension over field $K$, and let $J$ be any ideal of $D=D_{1} \times D_{2}$ (over $K$ ). If $Z_{1}$ and $Z_{2}$ are the centers of $D_{1}$ and $D_{2}$ respectively, then the center of $D / J$ is isomorphic (with respect to $K, Z_{1}$, or $Z_{2}$ ) to $Z / J_{z}$, where $Z=Z_{1} \times Z_{2}$ and $J_{z}=J \cap Z$. This enables one to prove structure theorems on algebras which aregenerated by every residue system modulo the radical. In particular, $D$ has the latter property if and only if its center has the same property. (Received October 14, 1954.)

\section{ANALYsis}

\section{P. C. Rosenbloom: An operational calculus.}

Let $L$ be a linear operator on a linear vector space such that analytic functions of $L$ can be defined with the usual properties. Then identities between ordinary analytic functions yield the corresponding identities between functions of $L$. These can of ten be interpreted as relations between solutions of different partial differential equations. Particular cases lead to the Riemann-Liouville fractional integrals, the Riesz potentials for elliptic equations, the work of Weinstein and his associates on the EulerPoisson-Darboux equation, and the John identities for spherical means. The formal calculations can be justified in part by Dunford's theory of the spectral resolution of operators. (Received November 12, 1954.)

\section{M. E. Shanks: Remarks on derivations and differentiability.}

Let $C^{0}(X)$ be the algebra of all continuous real functions on the space $X$, and if 
$X$ is a differentiable manifold of class $C^{k}$, let $C^{k}(X)$ be the functions with at least $k$ continuous derivatives. For class $C^{\infty}$ manifolds tangent vector fields are usually defined as derivations on $C^{\infty}(X)$ to $C^{\infty}(X)$. It is shown that the same definition applies in the case $C^{k}$, in that if $D$ is any derivation of $C^{k}(X)$ into $C^{r}(X), r<k$, then $D f$ $=\lambda^{i} \partial f / \partial x^{i}$ in the local coordinates with $\lambda^{i} \in C^{r}$. Something like converses to the above are obtained when $X=R=$ the real numbers. For example, if $C(R) \subset C^{0}(R)$ is an algebra over the reals which contains the polynomials and for which there is a derivation $D$ mapping $C(R)$ onto $C^{0}(R)$ such that $D x=1$ and $D f$ vanishes locally if and only if $f$ is locally constant, then $C(R)=C^{1}(R)$ and $D f=f^{\prime}$. Also, if $C \subset C^{0}(R)$ is a vector space closed under composition and $D$ is an operator from $C$ onto $C^{0}(R)$ satisfying the chain rule with local vanishing as above, then $C$ is an algebra, $C=C^{1}(R)$, and $D f=f^{\prime}$. Examples are given of algebras of nondifferentiable functions on which derivations onto $C^{0}(R)$ exist. Also examples of derivations on algebras of functions over spaces which are not manifolds. (Received October 14, 1954.)

\section{6t. Leonard Tornheim: Approximation by families of functions.}

An $n$-parameter family of functions $F$ on $[a, b]$ is a set of continuous functions for which exactly one member passes through each set of $n$ points on $[a, b]$ with distinct abscissas. It is a linear family if it is closed under the linear operations. For a given continuous function $g$ on $[a, b]$, let $M_{k}(f)=\int_{a}^{b}(f-g)^{2 k} d x$. Then $f_{k}$ in $F$ is a best $k$ approximant to $g$ if $M_{k}\left(f_{k}\right)=\mathrm{g} \cdot \mathrm{l} \cdot \mathrm{b} \cdot f \in F M_{k}(f)$. (1) A best $k$-approximant always exists. (2) It is unique if $F$ is linear but need not be in general. (3) Also it need not cross $g$. (4) $\operatorname{Lim}_{k \rightarrow \infty} f_{k}$ exists and equals $f_{\infty}$, the unique best approximant for which $\int_{a}^{b}|f-g| d x$ is a minimum. Properties (2) and (4) can be proved using the method of P6lya, C. R. Acad. Sci. Paris vol. 157 (1913) pp. 840-843. (Received October 13, 1954.)

\section{Applied Mathematics}

\section{7t. L. E. Payne: Inequalities for certain eigenvalues of a mem- brane. II.}

Let $D$ be a closed convex domain with boundary $C$ in the $x y$-plane and let $u(x, y)$ be a solution of the differential equation $\Delta u+k u=0$ in $D$ (where $k$ is a positive constant) and either (1) $u=0$ on $C$ or (2) $\partial u / \partial n=0$ on $C$. We denote the eigenvalues in case (1) by $\lambda_{1} \leqq \lambda_{2} \leqq \lambda_{3} \leqq \cdots$, and in case (2) by $0=\mu_{1} \leqq \mu_{2} \leqq \mu_{3} \leqq \cdots$. In a previous abstract (Bull. Amer. Math. Soc. Abstract 60-6-734) the author showed that $\mu_{3} \leqq \lambda_{1}$ $-1 / 2(\rho h)_{\max }$. This inequality is now improved to read (3) $\mu_{3} \leqq \lambda_{1}-1 /(\rho h)_{\max }$, and in addition the general inequality (4) $\mu_{n} \leqq \lambda_{n-2}$, which is valid for all $n \geqq 3$, is obtained. The research of this author was supported by the United States Air Force through the Office of Scientific Research. (Received September 27, 1954.)

\section{GeOMETRY}

\section{8t. P. C. Hammer: Planar curves of equivalent breadth.}

Earlier, the writer achieved an inversion of central symmetrization in the plane using geometrical methods (Bull. Amer. Math. Soc. Abstract 59-2-235). In this paper he uses analytical methods to achieve the same result and extends the results to include nonconvex curves of breadth equivalent to that of a given curve. In general, for any closed convex curve, the problem of determining all convex curves of equivalent breadth is reduced to that of determining all continuous increasing functions. (Received October 13, 1954.) 


\section{LOGIC AND FOUNDATIONS}

\section{J. W. Addison: Analogies in the Borel, Lusin, and Kleene} hierarchies. I.

Mostowski (Fund. Math. vol. 34 (1947) pp. 81-112) utilized an analogy with the projective hierarchy on the real line $R$ to develop the Kleene hierarchy on the set $N$ of natural numbers. An imperfection in this analogy was later found (Kleene, Neder. Akad. Wetensch. vol. 53 (1950) pp. 800-802). An analogy without this imperfection is shown to exist between the Borel hierarchy on $R$ and the Kleene hierarchy on $N$. Its cause is traced through the Borel hierarchy on $N^{N}$ (a $G_{\delta}$-subset of $R$ ) and the Kleene hierarchy on $N^{N}$. The finite Kleene hierarchy on $N^{N}$ is just the finite Borel hierarchy on $N^{N}$ with enumerable unions and intersections required to be effectively enumerable. Many parallel theorems, including separation theorems, about the Borel and Kleene hierarchies on $N^{N}$ are demonstrated by essentially the same proofs. Systematically changing a few symbols these proofs apply also to the Kleene hierarchy on $N$. Another less direct way of perfecting Mostowski's analogy is also exhibited. A purely recursive-function-theoretic result is proved by direct application of the Baire category theorem. Identifying $x(\in N)$ with the constant function $x$ the $n$th effective class of Baire-de la Vallee Poussin relative to $N$ (as a subspace of $N^{N}$ ) is just the class of sets of Post-Kleene degree $x$ for $n-1<x \leqq n$. The projective hierarchy corresponds to the Kleene function-quantifier hierarchy. (Recieved September 24, 1954.)

\section{Statistics and Probability}

\section{0t. H. W. Becker: Weighted factorial tables, an idea of Andre.}

Let $\sum_{R} P_{n, m}=P_{n}=n !, \sum m \cdot{ }_{R} P_{n, m}=P_{n}^{\prime}$. Andre classified permutations by alternations, $\mathrm{I} P_{n, m}$, Netto, Combinatorik (1927) pp. 105-112. Then ${ }_{\mathrm{I}} P_{n}^{\prime}=n !(2 n-1) / 3$. The number of permutations of $n$ letters with given letter $m$ fixed is ${ }_{\mathrm{II}} P_{n, m}=(n-1)$ ! For table, bibliographies, etc. of the number with $m$ inversions, ${ }_{\mathrm{III}} P_{n, m}$, see Piza, Math. Mag. vol. 21 (1948) p. 259, ibid. vol. 22 (1949) pp. 213-214, and Riordan, Proc. Amer. Math. Soc. vol. 2 (1951) pp. $429-432$. Then ${ }_{\mathrm{II}} P_{n}^{\prime}=\operatorname{mII} P_{n}^{\prime}=(n+1) ! / 2$; ${ }_{\text {II }} P_{n, m}$ and ${ }_{\text {III }} P_{n, m}$ are unequal, but isobaric. Call ${ }_{\mathrm{IV}} P_{n, m}$ the unsigned Stirling numbers of the first kind, Steffensen, Interpolation (1927) p. 57, which enumerate cycles of substitutions, Touchard, Acta Math. vol. 70 (1939) p. 243-297. Then ${ }_{\mathrm{Iv}} P_{n}^{\prime}={ }_{\mathrm{Iv}} P_{n+1,2}$ $=P(P+Q)^{n-1}$ where $Q_{0}=1$, otherwise $Q_{m}=(m-1)$ ! The number of permutations of $n$ letters, $m$ underanged, is $\mathrm{v} P_{n, m}=(n, m)(P-1)^{n-m}$. Then $\mathrm{v} P_{n}^{\prime}:=n !, n \geqq 1$; here $m$ is isobaric to unity, and $V=V(1,0)$. Let $V(r, s)$ denote weighting by $m+s$ of the $r$-place menages numbers enumerated for $r=2$ and 3 by Riordan and Kaplansky, Scripta Math. vol. 12 (1946) p. 124, and ibid. vol. $20(1954)$ p. 23. Then $\mathrm{v}(r, 8) P_{n}^{\prime}$ $=(r+s) \cdot n !, n \geqq r$, as far as known. Interpretation of $m \cdot{ }_{R} P_{n, m}$ is that each of the $m$ letters is starred in turn. If any combination is starred simultaneously, the wt. functions is $2^{m}$, the sum ${ }_{R} P_{n}^{\prime \prime}$. Then ${ }_{\mathrm{II}} P_{n}^{\prime \prime}=2\left(2^{n}-1\right)(n-1) ! ; \operatorname{III}_{n}^{\prime \prime}=2 K_{n}=(K+1)^{n}$ where $e^{t K}=1 /\left(2-e^{t}\right)$, Bull. Amer. Math. Soc. vol. $56(1950)$ p. $61 ; \operatorname{iv} P_{n}^{\prime \prime}=(n+1)$ !; v $P_{n}^{\prime \prime}=(P+1)^{n}$. (Received October 13,1954 .)

\section{TOPOLOGY}

\section{1t. A. L. Blakers: $A$ new Whitehead product for homotopy groups.}

Let $(X ; A, B)$ be a triad. A product is defined pairing $\pi_{p}(X ; A, B), p \geqq 3$, and $\pi_{q}(A \cap B), q \geqq 1$, to $\pi_{p+q-1}(X ; A, B)$. The definition is geometric and similar to those 
of previous generalizations of the Whitehead product. (See, for example, Ann. of Math. vol. 58 (1953) pp. 295-324.) The new product is bilinear when $p>3, q>1$. It is determined by the operators from $\pi_{1}(A \cap B)$ when $q=1$, and is shown to be related to the previous generalizations under the inclusion and boundary homomorphisms. (Received October 8, 1954.)

142t. M. E. Shanks: An example where the covering homotopy theorem fails.

Let $B$ be the Sierpinski universal curve, a 1-dimensional Peano space, and let $G$ be the group of all homeomorphisms of $B$ with the compact-open topology. R. D. Anderson has shown that $B$ is homogeneous (Bull. Amer. Math. Soc. Abstract 59-2-249). Then, if $H$ is the subgroup leaving a point invariant, $B$ is homeomorphic to the coset space $G / H$. It is shown that no arc in $B$ may be "lifted up" into $G$. Thus the covering homotopy theorem fails for maps of a point and $G$ is not a fiber space with fiber $H$, even though the base space is about as "nice" as possible. It follows from the proof that no locally compact subgroup of $G$ can act transitively on $B$. (Received October 14, 1954.)

J. W. T. Youngs, Associate Secretary 